\title{
Environmental Hazard Assessment of Jarosite Waste Using Batch Leaching Tests ${ }^{*}$
}

\author{
M. Kerolli - Mustafa, ${ }^{a,{ }^{* * *}}$ L. Ćurković, ${ }^{b}$ M. Ujević Bošnjak, ${ }^{c}$ and T. Rezićd \\ anternational Business College Mitrovica, Pajazit Boletini 22, \\ 40000 Mitrovica, Kosovo

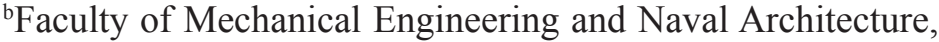 \\ University of Zagreb, Ivana Lučića 5, 10000 Zagreb, Croatia \\ ${ }^{\mathrm{c} C r o a t i a n}$ Institute of Public Health, Rockefellerova 7, \\ 10000 Zagreb, Croatia \\ ${ }^{d}$ Faculty of Food Technology and Biotechnology, \\ University of Zagreb, Pierottijeva 6, 10000 Zagreb, Croatia
}

doi: 10.15255/CABEQ.2017.1120

Jarosite waste samples from Trepça Zinc Industry in Kosovo were subjected to two batch leaching tests as an attempt to characterize the leaching behavior and mobility of minor and major elements of jarosite waste. To achieve this, deionized water and synthetic acidic rain leaching tests were employed. A two-step acidic treatment in microwave digestion system were used to dissolve jarosite waste samples, followed by determination of $\mathrm{Al}, \mathrm{Ag}, \mathrm{As}, \mathrm{Ba}, \mathrm{Cd}, \mathrm{Co}, \mathrm{Cr}, \mathrm{Cu}, \mathrm{Fe}, \mathrm{Mn}, \mathrm{Ni}, \mathrm{P}, \mathrm{Pb}, \mathrm{S}, \mathrm{Si}, \mathrm{Sr}$, and $\mathrm{Zn}$ by inductively coupled plasma optical emission spectrometry (ICP-OES). The validation of the procedure was performed by the analysis of two geochemical reference materials, S JR-3 and $\mathrm{S}$ Jsy-1. Two toxicity leaching tests revealed a high metal releasing of $\mathrm{Cd}, \mathrm{Cu}, \mathrm{Ni}, \mathrm{Mn}$, $\mathrm{Pb}, \mathrm{Zn}$, and As, and the metal release risk for these elements is still very high due the low $\mathrm{pH}$ and acid rain. The statistical analysis showed useful data information on the relationship between elements in jarosite samples in two different extraction conditions (deionized water and synthetic acid rain).

Key words:

leaching test, jarosite waste, deionized water, synthetic acid rain

\section{Introduction}

Industrial processes inevitably impact the environment on varying scales. There are different ways to measure pollutants when evaluating the environmental threat from a solid material of different industrial processes. Detailed characterization and leaching tests are required to assess the environmental stability of waste before any treatment possibility. Standard leaching tests are used to estimate the potential mobility of trace and major elements from the deposited waste. These tests are also used to assess the remediation options of contaminated sites, their range of efficiency and source of environmental risk characterization ${ }^{1}$. In general, leaching is a method that is used to remove soluble components from a solid matrix. In order to protect human health and groundwater quality from contamination by metals, over 100 leaching methods have been developed over the last few decades to

\footnotetext{
"Presented at the " $4^{\text {th }}$ International Symposium on Environmental Management - Towards Circular Economy (SEM2016), December 7 - 9, Zagreb, Croatia"

${ }^{* *}$ Corresponding author: m.kerolli@ibcmitrovica.eu
}

determine metal release and mobility in soils from waste materials ${ }^{2,3,4,5}$. These methods vary in mass of the samples, type and volume of leachant solution, method delivery, and time ${ }^{2}$. Leaching tests used in the laboratory are usually conducted using European Standard EN 12457-2:2005 (or German standard DIN 38414-S4) in deionized water ${ }^{4}$. Further, the Synthetic Precipitation Leaching Procedure (SPLP) ${ }^{6}$ test is currently used by several researchers to evaluate leaching of constituents from waste using dilute sulfuric/nitric acid solution ${ }^{2,6,8,9}$. Leaching of trace and major elements from jarosite waste is strongly affected by $\mathrm{pH}$, while the concentration of trace and major elements in the solution is the factor that classifies the jarosite as hazardous or non-hazardous waste. Researchers have suggested that waste material should be tested under conditions similar to their actual applications in re-use scenarios in order to achieve reliable results ${ }^{10}$. The framework of this study includes the comparison of two batch leaching methods using leachants of deionized $^{11}$ and synthetic acidic rain ${ }^{6}$. More specifically, the leaching tests for environmental hazard assessment of jarosite tailing waste of Trepça Zinc Industry, Kosovo were examined. 


\section{Materials and methods}

The jarosite waste used for testing was obtained from the jarosite tailing damps located in Mitrovica City, Kosovo. This waste is produced from the jarosite process that was used in Trepça Zinc Industry. In order to assess the leaching test, three jarosite samples in a depth of $0.20 \mathrm{~m}, 1 \mathrm{~m}$, and $2 \mathrm{~m}$ were taken. After sampling, the samples were airdried, sieved to a fraction less than $2 \mathrm{~mm}$, and stored in polypropylene containers until analyzed.

\section{Batch leaching tests}

The leaching potential of the elements present in the jarosite waste samples was determined using the batch leaching test in deionized water according to HRN EN 12457-2 $2^{11}$, as well as the leaching test in synthetic acid rain according to the modification of Synthetic Precipitation Leaching Procedure (SPLP) Method 1312:19946.

\section{Chemical analysis methodology}

Total metal content of trace and major metals was determined using microwave digestion method (MARSX XP1500 Microwave Digestion System, CEM, SAD). Microwave digestion of jarosite waste samples was carried out in two stages with the use of reagents $\mathrm{HCl}, \mathrm{HNO}_{3}, \mathrm{HF}$ and $\mathrm{H}_{3} \mathrm{BO}_{3}{ }^{12,13}$. Certified reference materials, S JR-3 and S Jsy-1 (MBH Analytical LTD), were analyzed in order to test the accuracy of the applied method for determination of total metal concentrations in the investigated jarosite samples. The concentrations of constituents (Al, Ag, As, Ba, Cd, Co, Cr, Cu, Fe, Mn, Ni, P, Pb, $\mathrm{S}, \mathrm{Si}, \mathrm{Sr}$, and $\mathrm{Zn}$ ) in the leachates were determined by Thermo Inductive Coupled Plasma Optical Emission Spectrometer (ICP-OES, IRIS Interpid II XSP). The concentration of constituents in leachates was compared with the leaching limit values of the decision 2003/33/EC ${ }^{14}$. The electrical conductivity and $\mathrm{pH}$ were measured using conductivity meter (MA 5964 Iskra, Croatia) and $\mathrm{pH}$ meter (FE20, Mettler Toledo, Switzerland). Both these pieces of equipment were calibrated using standard solution before measurements. The measurements for all parameters were conducted simultaneously or different times of 1 day, 7 days, 14 days, and 21 days. Quality assurance of the leaching process was achieved with the application of US EPA QA/QS protocol ${ }^{7}$ and the use of blank method for every batch of samples.

\section{Statistical analysis}

The influence of the variables, such as concentration, depth, and time of the extraction, were investigated using the mathematical and statistical analysis method of Response Surface Methodology (RSM). The statistical analyses were performed using Statistica (data analysis software system), version 10 and software Excel 2010. Statistical significance of the variables was determined at the $5 \%$ probability level $(p<0.05)$. Statistical significance was determined by Student's $t$-test and polynomial equations describing the responses of variations. The three-dimensional surface was plotted for the results of leaching tests in deionized water and synthetic acid rain. The interaction and the effects during the leaching process between concentration, depth, and leaching time were analyzed using Paretto charts. Moreover, the mathematical models for description of responsive variables of the depth and time in the concentration of each leachant ratio were established.

\section{Results and discussions}

\section{Total metal content}

The results of total metal content determined in accordance with the described microwave digestion method are presented in Table 1 for the samples of depth $0.20 \mathrm{~m}$ (Sample 1), $1 \mathrm{~m}$ (Sample 2), and $2 \mathrm{~m}$ (Sample 3). In samples 1, 2, and 3, respectively, $\mathrm{Fe}$ in the form of $\mathrm{Fe}_{2} \mathrm{O}_{3}$ had the highest content (44.94 wt.\%, 45.14 wt.\% and 44.37 wt.\%) among all other elements. $\mathrm{SiO}_{2}$ is also an important component with 6.31 wt.\%, 5.92 wt.\% and 4.20 wt.\%. Zn had the highest content among all heavy metals (10.91 wt.\%, 5.24 wt.\%, 1.33 wt.\%), followed by $\mathrm{Pb}$ (7.51 wt.\%, 5.17 wt.\%, 6.93 wt.\%), where $\mathrm{Pb}$ in sample 1 had the highest concentration, and $\mathrm{Cu}$ ( 0.97 wt. $\%, 0.679$ wt. $\%, 0.40$ wt.\%). The concentration of other metals ranged as follows: Ag (133.82151.87), Ba (370.62-578.90), Co (9.79-30.3), Cd (418.25-2308.69), Cr (79.30-416.59), Mn (1905.076391.71), Ni (6.13-93.50), Sr (156.20-200.36), and As (200.36-5075.53) $\mathrm{mg} \mathrm{kg}^{-1}$ jarosite waste. The concentration of heavy metals varied from $0.20 \mathrm{~m}$ to $2 \mathrm{~m}$ sample depths. In all cases, the difference was higher than $40 \%$ from $2 \mathrm{~m}$ up to $0.20 \mathrm{~m}$ depth, indicating a high degree of concentration of available heavy metals in the surface of jarosite tailing damp.

\section{$\mathrm{pH}$ values and electrical conductivity (EC) of leachates}

Fig. 1 shows the changes in $\mathrm{pH}$ values during contact time of jarosite waste with deionized water and synthetic acid rain leaching tests. The initial $\mathrm{pH}$ value in deionized water was 5.53 , while the $\mathrm{pH}$ values in the period of 1 day, 7 days, 14 days, and 21 days ranged from 3.96 to 3.66 for sample 1, 3.68 
Table 1 - Total metal content in jarosite waste samples $(n=3)$

\begin{tabular}{l|c|c|c}
\hline \multicolumn{1}{c|}{ Element } & $\begin{array}{c}\text { Sample 1 } \\
(0.2 \mathrm{~m} \text { depth }) \\
\text { mean } \pm \sigma\end{array}$ & $\begin{array}{c}\text { Sample 2 } \\
(1 \mathrm{~m} \text { depth }) \\
\text { mean } \pm \sigma\end{array}$ & $\begin{array}{c}\text { Sample 3 } \\
(2 \mathrm{~m} \text { depth }) \\
\text { mean } \pm \sigma\end{array}$ \\
\hline $\mathrm{Al}_{2} \mathrm{O}_{3}$, wt. \% & $1.42 \pm 0.02$ & $1.09 \pm 0.01$ & $0.62 \pm 0.001$ \\
$\mathrm{Fe}_{2} \mathrm{O}_{3}$, wt. \% & $44.94 \pm 0.05$ & $45.14 \pm 0.54$ & $44.37 \pm 0.48$ \\
$\mathrm{SiO}_{2}$, wt. \% & $6.31 \pm 0.7$ & $5.92 \pm 0.6$ & $4.20 \pm 0.2$ \\
$\mathrm{Zn}$, wt. \% & $10.91 \pm 0.02$ & $5.24 \pm 0.2$ & $1.33 \pm 0.01$ \\
$\mathrm{~Pb}$, wt. \% & $7.51 \pm 0.13$ & $5.17 \pm 0.04$ & $6.93 \pm 0.04$ \\
$\mathrm{Cu}, \mathrm{wt.} \%$ & $0.97 \pm 0.01$ & $0.679 \pm 0.01$ & $0.40 \pm 0.00$ \\
$\mathrm{Ag}, \mathrm{mg} \mathrm{kg}{ }^{-1}$ & $133.82 \pm 11.82$ & $139.77 \pm 6.29$ & $151.87 \pm 1.38$ \\
$\mathrm{Ba}, \mathrm{mg} \mathrm{kg}^{-1}$ & $578.90 \pm 194.73$ & $428.12 \pm 121.03$ & $370.62 \pm 64.49$ \\
$\mathrm{Co}, \mathrm{mg} \mathrm{kg}^{-1}$ & $30.38 \pm 0.27$ & $27.55 \pm 0.48$ & $9.79 \pm 0.65$ \\
$\mathrm{Cd}, \mathrm{mg} \mathrm{kg}^{-1}$ & $2308.69 \pm 24.47$ & $1359.03 \pm 9.48$ & $418.25 \pm 2.59$ \\
$\mathrm{Cr}, \mathrm{mg} \mathrm{kg}$ & $416.59 \pm 14.35$ & $96.71 \pm 0.80$ & $79.30 \pm 1.29$ \\
$\mathrm{Mn}, \mathrm{mg} \mathrm{kg}^{-1}$ & $6391.71 \pm 84.06$ & $3741.27 \pm 58.43$ & $1905.07 \pm 20.19$ \\
$\mathrm{Ni}, \mathrm{mg} \mathrm{kg}^{-1}$ & $93.50 \pm 1.10$ & $53.00 \pm 0.45$ & $6.13 \pm 2.89$ \\
$\mathrm{Sr}, \mathrm{mg} \mathrm{kg}^{-1}$ & $156.20 \pm 2.01$ & $177.84 \pm 0.04$ & $200.36 \pm 0.57$ \\
$\mathrm{As}, \mathrm{mg} \mathrm{kg}^{-1}$ & $5075.53 \pm 5.52$ & $3014.85 \pm 0.00$ & $2534.93 \pm 0.64$ \\
\hline
\end{tabular}

to 3.59 for sample 2, and 3.57 to 3.52 for sample 3 . The $\mathrm{pH}$ value of leachates ranged stably from 3.96 to 3.52 , which indicated that the jarosite waste had high acidic buffering capacity. The $\mathrm{pH}$ value in the acid rain condition during the leaching process changed negligibly. This complies with the conclusion of Min et al. ${ }^{14}$, that, under these conditions, the leaching of heavy metals from waste was a longterm process that accumulated in the environment. Fig. 2 shows the changes in electrical conductivity (EC) during time in contact of jarosite waste. According to the obtained results, the electrical conductivity (EC) increases very fast in a short period from the initial value for both leaching tests (in deionized water and synthetic acid rain). Higher values of the electrical conductivity were obtained for the leaching with the synthetic acid rain.

\section{Leaching tests of jarosite samples with deionized water and synthetic acid rain}

The results of the leaching jarosite samples in deionized water and synthetic acid rain are presented in Tables 2 and 3. As may be seen, constituents such as $\mathrm{Zn}, \mathrm{S}$, and Mn presented high mobility. As many studies have shown, the release potential of constituents from jarosite waste materials may vary depending on various physicochemical factors, such as $\mathrm{pH}, \mathrm{L} / \mathrm{S}$ (liquid/solid) ratio, the matrix of solid waste and the leaching type ${ }^{16}$. According to the results shown in Table 2, the highest values of $\mathrm{Al}, \mathrm{Cd}$,
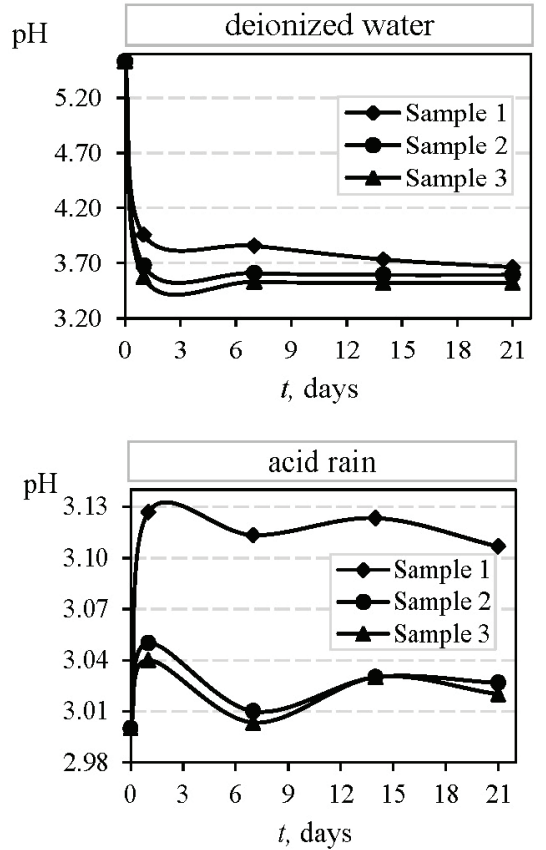

Fig. $1-p H$ values of leachates in deionized water and synthetic acid rain in deionized water as leachant
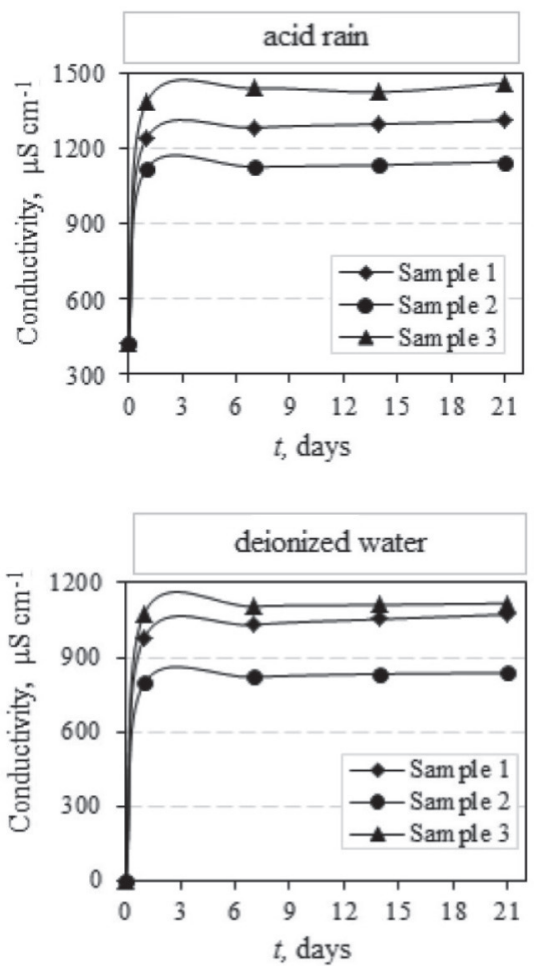

Fig. 2 - Electrical conductivity (EC) of leachates in deionized water and synthetic acid rain

$\mathrm{Cu}, \mathrm{Fe}, \mathrm{Pb}$, and Si were $212.11 \mathrm{mg} \mathrm{kg}^{-1}, 69.95 \mathrm{mg} \mathrm{kg}^{-1}$, $139.30 \mathrm{mg} \mathrm{kg}^{-1}, 39.16 \mathrm{mg} \mathrm{kg}^{-1}, 168.21 \mathrm{mg} \mathrm{kg}^{-1}$, and $194.09 \mathrm{mg} \mathrm{kg}^{-1}$, respectively. These elements showed the highest value on the 21-day leaching time, except Mn, which showed the highest value of leaching after 1 day. 
Table 2 -Average concentration of major and minor elements in samples leached with deionized water

\begin{tabular}{|c|c|c|c|c|c|}
\hline \multirow{3}{*}{ Element } & \multirow{3}{*}{ Sample } & \multicolumn{4}{|c|}{$t$, days } \\
\hline & & 1 & 7 & 14 & 21 \\
\hline & & $\begin{array}{c}\mathrm{mg} \mathrm{kg}{ }^{-1} \\
\text { mean } \pm \sigma\end{array}$ & $\begin{array}{c}\mathrm{mg} \mathrm{kg}{ }^{-1} \\
\text { mean } \pm \sigma\end{array}$ & $\begin{array}{c}\mathrm{mg} \mathrm{kg}{ }^{-1} \\
\text { mean } \pm \sigma\end{array}$ & $\begin{array}{c}\mathrm{mg} \mathrm{kg}{ }^{-1} \\
\text { mean } \pm \sigma\end{array}$ \\
\hline \multirow{3}{*}{$\mathrm{Ag}$} & 1 & $0.11 \pm 0.07$ & $0.09 \pm 0.03$ & $0.03 \pm 0.05$ & $0.13 \pm 0.01$ \\
\hline & 2 & $0.36 \pm 0.22$ & $0.29 \pm 0.10$ & $0.11 \pm 0.04$ & $0.63 \pm 0.39$ \\
\hline & 3 & $0.07 \pm 0.04$ & $0.06 \pm 0.02$ & $0.10 \pm 0.04$ & $0.32 \pm 0.09$ \\
\hline \multirow{3}{*}{$\mathrm{Al}$} & 1 & $82.24 \pm 5.00$ & $77.68 \pm 1.29$ & $78.82 \pm 0.97$ & $97.82 \pm 3.67$ \\
\hline & 2 & $197.60 \pm 6.02$ & $191.32 \pm 7.26$ & $184.24 \pm 1.29$ & $212.11 \pm 5.32$ \\
\hline & 3 & $181.95 \pm 7.82$ & $170.08 \pm 1.02$ & $167.65 \pm 0.71$ & $194.27 \pm 1.07$ \\
\hline \multirow{3}{*}{$\mathrm{Ba}$} & 1 & $0.13 \pm 0.11$ & $0.03 \pm 0.06$ & $0.06 \pm 0.03$ & $0.28 \pm 0.06$ \\
\hline & 2 & $0.77 \pm 0.11$ & $0.62 \pm 0.05$ & $0.64 \pm 0.04$ & $0.76 \pm 0.15$ \\
\hline & 3 & $0.55 \pm 0.05$ & $0.36 \pm 0.03$ & $0.46 \pm 0.03$ & $0.70 \pm 0.09$ \\
\hline \multirow{3}{*}{$\mathrm{Cd}$} & 1 & $51.67 \pm 3.89$ & $51.06 \pm 1.09$ & $50.67 \pm 0.26$ & $56.98 \pm 0.31$ \\
\hline & 2 & $62.68 \pm 1.17$ & $63.21 \pm 1.71$ & $62.82 \pm 0.81$ & $69.95 \pm 0.69$ \\
\hline & 3 & $58.60 \pm 2.15$ & $59.51 \pm 0.61$ & $59.17 \pm 0.49$ & $66.06 \pm 1.06$ \\
\hline \multirow{3}{*}{$\mathrm{Co}$} & 1 & $0.67 \pm 0.02$ & $0.71 \pm 0.02$ & $0.69 \pm 0.02$ & $0.92 \pm 0.04$ \\
\hline & 2 & $1.26 \pm 0.03$ & $1.21 \pm 0.07$ & $1.22 \pm 0.03$ & $1.54 \pm 0.02$ \\
\hline & 3 & $1.28 \pm 0.09$ & $1.30 \pm 0.03$ & $1.26 \pm 0.02$ & $1.59 \pm 0.06$ \\
\hline \multirow{3}{*}{$\mathrm{Cr}$} & 1 & $0.03 \pm 0.04$ & - & - & - \\
\hline & 2 & $0.13 \pm 0.02$ & - & - & $0.08 \pm 0.05$ \\
\hline & 3 & $0.30 \pm 0.07$ & $0.06 \pm 0.03$ & $0.07 \pm 0.07$ & $0.18 \pm 0.05$ \\
\hline \multirow{3}{*}{$\mathrm{Cu}$} & 1 & $59.37 \pm 3.51$ & $62.52 \pm 1.66$ & $61.58 \pm 1.06$ & $71.42 \pm 1.47$ \\
\hline & 2 & $116.89 \pm 6.60$ & $115.23 \pm 3.56$ & $109.06 \pm 1.64$ & $124.77 \pm 3.30$ \\
\hline & 3 & $122.63 \pm 6.00$ & $32.38 \pm 9.77$ & $121.24 \pm 0.54$ & $139.30 \pm 1.50$ \\
\hline \multirow{3}{*}{$\mathrm{Fe}$} & 1 & $27.04 \pm 9.97$ & $22.55 \pm 4.53$ & $14.71 \pm 10.68$ & $10.14 \pm 2.23$ \\
\hline & 2 & $12.71 \pm 5.87$ & $3.69 \pm 3.26$ & $6.12 \pm 1.25$ & $27.53 \pm 14.69$ \\
\hline & 3 & $27.87 \pm 3.22$ & $39.16 \pm 2.10$ & $32.51 \pm 2.90$ & $42.37 \pm 8.93$ \\
\hline \multirow{3}{*}{$\mathrm{Mn}$} & 1 & $373.70 \pm 31.58$ & $363.89 \pm 7.97$ & $14.71 \pm 10.68$ & $10.14 \pm 2.23$ \\
\hline & 2 & $902.87 \pm 18.02$ & $888.39 \pm 21.88$ & $6.12 \pm 1.25$ & $27.53 \pm 14.69$ \\
\hline & 3 & $711.29 \pm 25.79$ & $32.38 \pm 9.77$ & $32.51 \pm 2.90$ & $42.37 \pm 8.93$ \\
\hline \multirow{3}{*}{$\mathrm{Ni}$} & 1 & $2.71 \pm 0.77$ & $2.36 \pm 0.05$ & $2.66 \pm 0.28$ & $3.97 \pm 0.52$ \\
\hline & 2 & $3.32 \pm 0.24$ & $2.61 \pm 0.14$ & $2.72 \pm 0.12$ & $3.31 \pm 0.11$ \\
\hline & 3 & $2.96 \pm 0.75$ & $2.42 \pm 0.06$ & $2.38 \pm 0.06$ & $2.81 \pm 0.07$ \\
\hline \multirow{3}{*}{$\mathrm{P}$} & 1 & $1.13 \pm 0.36$ & $1.33 \pm 0.72$ & $0.66 \pm 0.70$ & $0.28 \pm 0.38$ \\
\hline & 2 & $1.60 \pm 0.43$ & $1.65 \pm 0.07$ & $1.00 \pm 0.19$ & $0.77 \pm 0.55$ \\
\hline & 3 & $0.98 \pm 0.24$ & $1.13 \pm 0.14$ & $0.85 \pm 0.68$ & $0.47 \pm 0.36$ \\
\hline \multirow{3}{*}{$\mathrm{Pb}$} & 1 & $141.54 \pm 5.79$ & $158.52 \pm 2.50$ & $163.26 \pm 2.53$ & $168.21 \pm 8.35$ \\
\hline & 2 & $99.20 \pm 6.47$ & $119.15 \pm 2.72$ & $110.59 \pm 4.14$ & $146.33 \pm 22.32$ \\
\hline & 3 & $111.64 \pm 10.5$ & $132.69 \pm 8.88$ & $126.69 \pm 7.92$ & $139.30 \pm 7.41$ \\
\hline \multirow{3}{*}{$\mathrm{S}$} & 1 & $9667.37 \pm 434$ & $10159.97 \pm 209$ & $9968.83 \pm 219$ & $11956.19 \pm 68.6$ \\
\hline & 2 & $7317.88 \pm 83$ & $7761.42 \pm 346$ & $7575.21 \pm 186$ & $9199.62 \pm 41.52$ \\
\hline & 3 & $10179.06 \pm 291$ & $10466.74 \pm 247$ & $7454.01 \pm 5567$ & $12332.77 \pm 174$ \\
\hline \multirow{3}{*}{$\mathrm{Si}$} & 1 & $31.82 \pm 0.67$ & $118.76 \pm 1.57$ & $153.95 \pm 4.20$ & $194.09 \pm 2.65$ \\
\hline & 2 & $21.35 \pm 0.52$ & $78.91 \pm 2.82$ & $100.56 \pm 3.76$ & $128.39 \pm 2.87$ \\
\hline & 3 & $22.20 \pm 1.25$ & $77.96 \pm 0.98$ & $407.40 \pm 526.15$ & $129.51 \pm 6.64$ \\
\hline \multirow{3}{*}{$\mathrm{Sr}$} & 1 & $10.61 \pm 0.28$ & $11.70 \pm 0.13$ & $12.53 \pm 0.19$ & $14.49 \pm 0.38$ \\
\hline & 2 & $20.33 \pm 0.85$ & $24.88 \pm 0.68$ & $25.03 \pm 0.69$ & $29.49 \pm 0.21$ \\
\hline & 3 & $17.78 \pm 0.74$ & $19.68 \pm 0.05$ & $20.52 \pm 0.47$ & $24.11 \pm 0.44$ \\
\hline \multirow{3}{*}{$\mathrm{Zn}$} & 1 & $5601.21 \pm 49$ & $5617.90 \pm 38$ & $4109.97 \pm 2684$ & $3331.20 \pm 25.76$ \\
\hline & 2 & $4886.89 \pm 87$ & $4893.50 \pm 26$ & $4883.12 \pm 61.08$ & $2897.89 \pm 4.61$ \\
\hline & 3 & $4694.21 \pm 148$ & $4807.21 \pm 78$ & $4684.80 \pm 38.98$ & $2787.88 \pm 47.86$ \\
\hline & 1 & $0.33 \pm 0.07$ & $0.67 \pm 0.00$ & $2.04 \pm 0.00$ & $2.84 \pm 0.19$ \\
\hline As & 2 & $0.53 \pm 0.00$ & $0.10 \pm 0.00$ & $0.23 \pm 0.00$ & $0.54 \pm 0.23$ \\
\hline & 3 & $0.10 \pm 0.00$ & $0.08 \pm 0.00$ & $0.19 \pm 0.00$ & $0.28 \pm 0.08$ \\
\hline
\end{tabular}


Table 3 -Average concentration of major and minor elements in samples leached with synthetic acid rain leaching tests

\begin{tabular}{|c|c|c|c|c|c|}
\hline \multirow{3}{*}{ Element } & \multirow{3}{*}{ Sample } & \multicolumn{4}{|c|}{$t$, days } \\
\hline & & 1 & 7 & 14 & 21 \\
\hline & & $\begin{array}{c}\mathrm{mg} \mathrm{kg}^{-1} \\
\text { mean } \pm \sigma\end{array}$ & $\begin{array}{c}\mathrm{mg} \mathrm{kg}^{-1} \\
\text { mean } \pm \sigma\end{array}$ & $\begin{array}{c}m g \mathrm{~kg}^{-1} \\
\text { mean } \pm \sigma\end{array}$ & $\begin{array}{c}\mathrm{mg} \mathrm{kg}^{-1} \\
\text { mean } \pm \sigma\end{array}$ \\
\hline \multirow{3}{*}{$\mathrm{Ag}$} & 1 & $0.52 \pm 0.42$ & $0.19 \pm 0.06$ & $0.23 \pm 0.11$ & $0.95 \pm 0.69$ \\
\hline & 2 & $0.38 \pm 0.09$ & $0.56 \pm 0.16$ & $0.43 \pm 0.07$ & $1.00 \pm 1.13$ \\
\hline & 3 & $0.34 \pm 0.07$ & $0.47 \pm 0.12$ & $0.46 \pm 0.21$ & $0.59 \pm 0.03$ \\
\hline \multirow{3}{*}{$\mathrm{Al}$} & 1 & $129.17 \pm 4.70$ & $128.92 \pm 5.09$ & $130.52 \pm 9.25$ & $103.65 \pm 86.26$ \\
\hline & 2 & $217.85 \pm 11.18$ & $209.49 \pm 4.54$ & $208.07 \pm 15.99$ & $167.50 \pm 142.18$ \\
\hline & 3 & $198.17 \pm 10.29$ & $192.03 \pm 6.74$ & $190.04 \pm 4.00$ & $217.57 \pm 4.30$ \\
\hline \multirow{3}{*}{$\mathrm{Ba}$} & 1 & $0.10 \pm 0.03$ & $0.02 \pm 0.01$ & $0.07 \pm 0.02$ & $0.13 \pm 0.13$ \\
\hline & 2 & $0.60 \pm 0.04$ & $0.46 \pm 0.03$ & $0.59 \pm 0.06$ & $0.49 \pm 0.43$ \\
\hline & 3 & $0.57 \pm 0.08$ & $0.34 \pm 0.02$ & $0.49 \pm 0.01$ & $0.75 \pm 0.30$ \\
\hline \multirow{3}{*}{$\mathrm{Cd}$} & 1 & $51.54 \pm 1.13$ & $51.51 \pm 1.60$ & $52.44 \pm 3.38$ & $39.04 \pm 32.93$ \\
\hline & 2 & $63.79 \pm 2.82$ & $62.11 \pm 0.21$ & $61.35 \pm 3.18$ & $47.82 \pm 40.74$ \\
\hline & 3 & $61.00 \pm 1.53$ & $60.50 \pm 1.35$ & $59.19 \pm 1.01$ & $66.43 \pm 0.88$ \\
\hline \multirow{3}{*}{$\mathrm{Co}$} & 1 & $0.72 \pm 0.03$ & $0.72 \pm 0.04$ & $0.71 \pm 0.06$ & $0.63 \pm 0.51$ \\
\hline & 2 & $1.25 \pm 0.08$ & $1.21 \pm 0.03$ & $1.19 \pm 0.08$ & $1.01 \pm 0.85$ \\
\hline & 3 & $1.33 \pm 0.06$ & $1.31 \pm 0.03$ & $1.29 \pm 0.04$ & $1.56 \pm 0.03$ \\
\hline \multirow{3}{*}{$\mathrm{Cr}$} & 1 & $0.25 \pm 0.04$ & $0.24 \pm 0.03$ & $0.26 \pm 0.03$ & $0.28 \pm 0.20$ \\
\hline & 2 & $0.36 \pm 0.03$ & $0.29 \pm 0.02$ & $0.30 \pm 0.04$ & $0.31 \pm 0.24$ \\
\hline & 3 & $0.47 \pm 0.06$ & $0.41 \pm 0.03$ & $0.35 \pm 0.04$ & $0.45 \pm 0.04$ \\
\hline \multirow{3}{*}{$\mathrm{Cu}$} & 1 & $78.93 \pm 3.77$ & $84.59 \pm 4.01$ & $86.35 \pm 6.26$ & $68.98 \pm 56.44$ \\
\hline & 2 & $133.67 \pm 8.03$ & $126.70 \pm 1.69$ & $126.48 \pm 9.83$ & $99.33 \pm 83.37$ \\
\hline & 3 & $154.62 \pm 11.28$ & $149.47 \pm 3.81$ & $146.35 \pm 3.84$ & $165.99 \pm 3.74$ \\
\hline \multirow{3}{*}{$\mathrm{Fe}$} & 1 & $55.30 \pm 13.28$ & $49.31 \pm 6.62$ & $31.65 \pm 2.05$ & $36.86 \pm 28.40$ \\
\hline & 2 & $40.06 \pm 3.77$ & $37.82 \pm 13.77$ & $52.04 \pm 20.31$ & $34.04 \pm 29.43$ \\
\hline & 3 & $93.74 \pm 5.33$ & $71.25 \pm 3.04$ & $85.54 \pm 10.16$ & $86.64 \pm 11.96$ \\
\hline \multirow{3}{*}{$\mathrm{Mn}$} & 1 & $352.31 \pm 9.26$ & $354.76 \pm 11.62$ & $357.13 \pm 22.85$ & $267.14 \pm 218.72$ \\
\hline & 2 & $812.10 \pm 31.92$ & $844.50 \pm 4.39$ & $832.91 \pm 47.79$ & $639.67 \pm 544.45$ \\
\hline & 3 & $711.74 \pm 23.53$ & $703.48 \pm 18.83$ & $690.40 \pm 14.53$ & $758.61 \pm 10.38$ \\
\hline \multirow{3}{*}{$\mathrm{Ni}$} & 1 & $3.00 \pm 0.58$ & $2.38 \pm 0.03$ & $2.52 \pm 0.19$ & $1.95 \pm 1.66$ \\
\hline & 2 & $3.02 \pm 0.39$ & $2.59 \pm 0.13$ & $2.42 \pm 0.11$ & $2.41 \pm 2.15$ \\
\hline & 3 & $3.19 \pm 0.55$ & $2.75 \pm 0.24$ & $2.38 \pm 0.03$ & $2.96 \pm 0.10$ \\
\hline \multirow{3}{*}{$\mathrm{P}$} & 1 & $1.16 \pm 0.09$ & $0.61 \pm 0.51$ & $0.68 \pm 0.57$ & $0.88 \pm 0.17$ \\
\hline & 2 & $1.34 \pm 0.75$ & $0.95 \pm 0.52$ & $1.04 \pm 0.26$ & $0.98 \pm 1.01$ \\
\hline & 3 & $1.08 \pm 0.75$ & $1.16 \pm 0.32$ & $0.72 \pm 0.80$ & $1.68 \pm 0.35$ \\
\hline \multirow{3}{*}{$\mathrm{Pb}$} & 1 & $160.00 \pm 4.55$ & $159.52 \pm 11.35$ & $162.07 \pm 1.92$ & $135.38 \pm 113.54$ \\
\hline & 2 & $120.86 \pm 0.90$ & $127.72 \pm 5.65$ & $134.31 \pm 16.13$ & $101.34 \pm 84.66$ \\
\hline & 3 & $125.24 \pm 6.48$ & $137.24 \pm 1.44$ & $137.97 \pm 4.33$ & $154.60 \pm 7.79$ \\
\hline \multirow{3}{*}{$\mathrm{S}$} & 1 & $11249.93 \pm 322.45$ & $11112.74 \pm 422.86$ & $11189.72 \pm 736.41$ & $12937.33 \pm 121.76$ \\
\hline & 2 & $8695.83 \pm 380.91$ & $8351.59 \pm 128.96$ & $8253.54 \pm 467.33$ & $9951.62 \pm 331.73$ \\
\hline & 3 & $11651.58 \pm 545.22$ & $11611.46 \pm 158.24$ & $11083.29 \pm 328.05$ & $13274.34 \pm 199.02$ \\
\hline \multirow{3}{*}{$\mathrm{Si}$} & 1 & $18.99 \pm 0.65$ & $69.46 \pm 3.88$ & $96.52 \pm 7.08$ & $123.16 \pm 1.37$ \\
\hline & 2 & $17.79 \pm 1.25$ & $55.74 \pm 0.84$ & $78.18 \pm 5.01$ & $107.03 \pm 13.45$ \\
\hline & 3 & $17.77 \pm 1.14$ & $61.08 \pm 1.63$ & $81.11 \pm 1.71$ & $101.75 \pm 1.84$ \\
\hline \multirow{3}{*}{$\mathrm{Sr}$} & 1 & $8.91 \pm 0.32$ & $10.50 \pm 0.12$ & $11.65 \pm 0.37$ & $9.38 \pm 7.97$ \\
\hline & 2 & $15.15 \pm 0.36$ & $17.12 \pm 0.16$ & $17.46 \pm 0.82$ & $72.35 \pm 44.38$ \\
\hline & 3 & $12.69 \pm 0.54$ & $15.03 \pm 0.21$ & $15.64 \pm 0.17$ & $71.30 \pm 46.20$ \\
\hline \multirow{3}{*}{$\mathrm{Zn}$} & 1 & $5494.14 \pm 16.14$ & $5490.92 \pm 92.84$ & $5619.06 \pm 223.09$ & $3422.29 \pm 48.60$ \\
\hline & 2 & $4901.83 \pm 110.16$ & $4756.44 \pm 26.53$ & $4679.24 \pm 144.79$ & $2933.39 \pm 133.04$ \\
\hline & 3 & $4746.49 \pm 78.19$ & $4747.84 \pm 95.63$ & $4642.41 \pm 65.81$ & $2834.81 \pm 26.92$ \\
\hline & 1 & $0.45 \pm 0.00$ & $0.86 \pm 0.00$ & $1.57 \pm 0.34$ & $2.79 \pm 2.43$ \\
\hline As & 2 & $0.12 \pm 0.00$ & $0.14 \pm 0.00$ & $0.30 \pm 0.00$ & $0.38 \pm 0.44$ \\
\hline & 3 & $0.35 \pm 0.00$ & $0.21 \pm 0.00$ & $0.06 \pm 0.00$ & $0.43 \pm 0.12$ \\
\hline
\end{tabular}


a

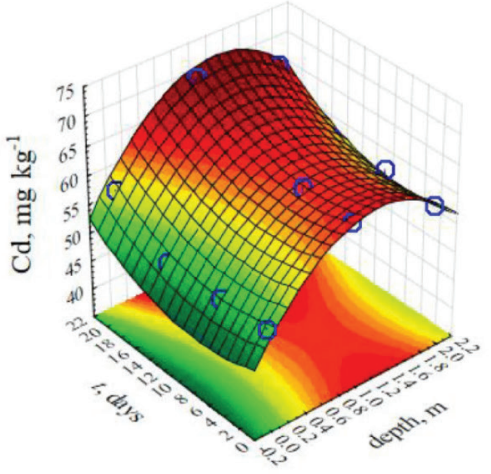

a

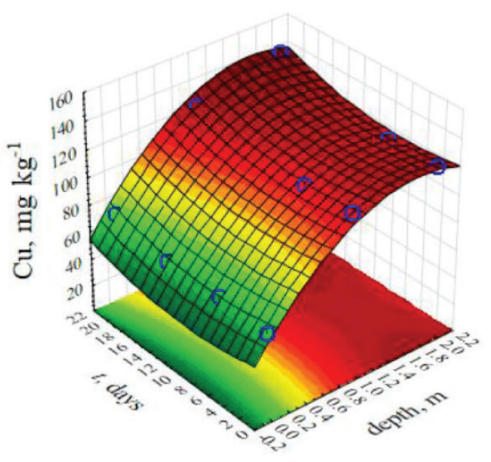

a

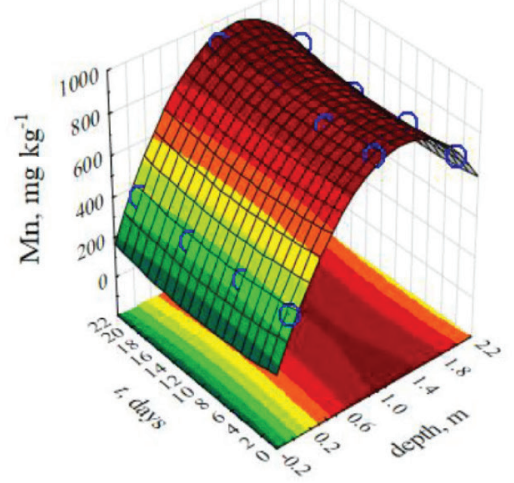

a

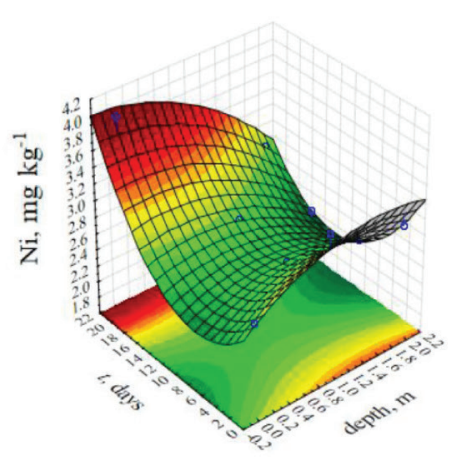

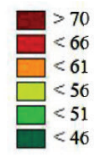

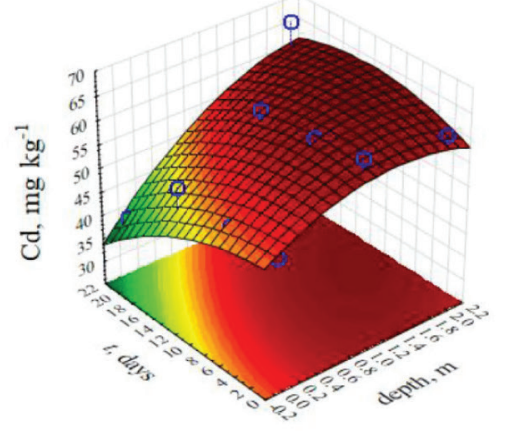

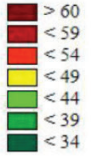

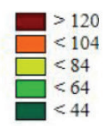

b

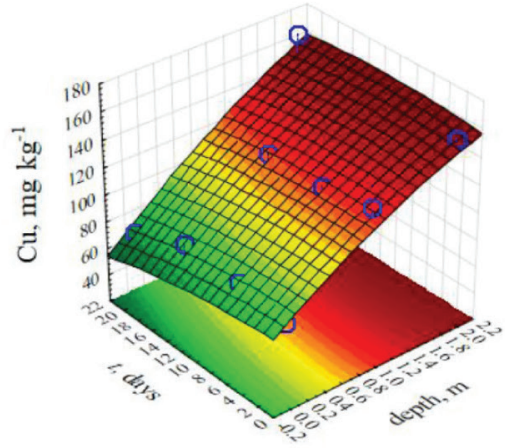

Q $>160$

$\square_{<136}^{<156}$

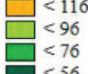

$<56$
$>>800$
$<<00$
$\square<600$
$<<200$
$<200$

b

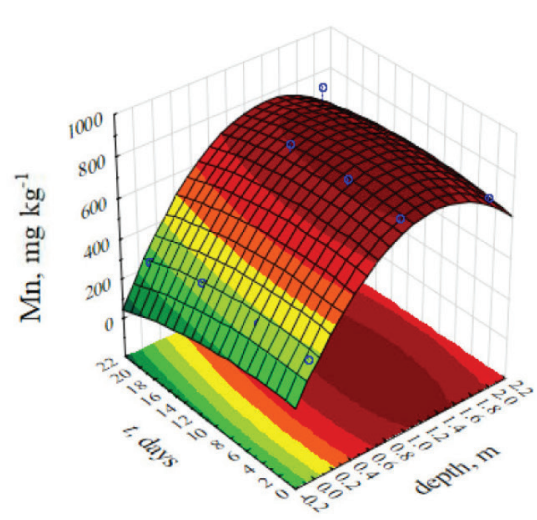

b
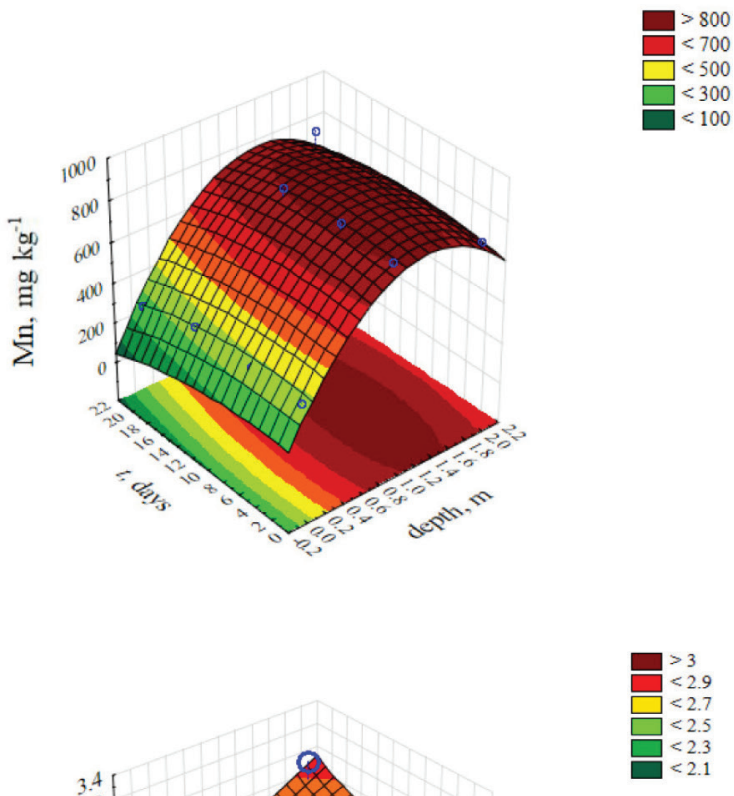

Fig. 3 - Continued on next page...
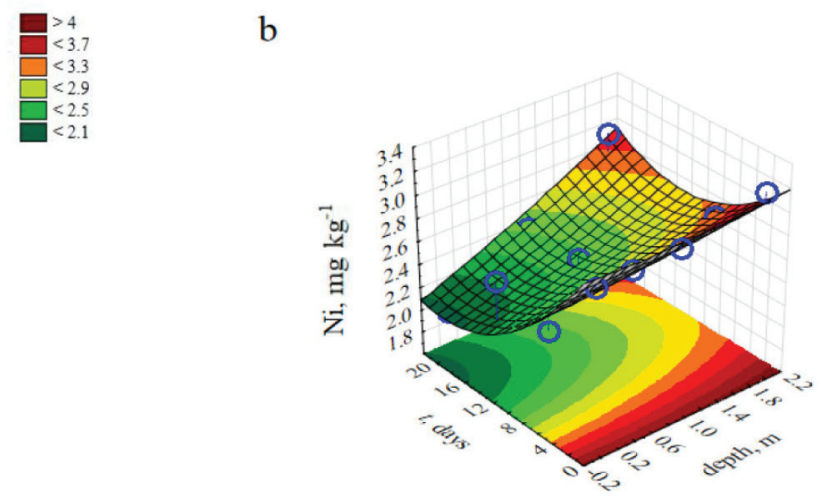
a

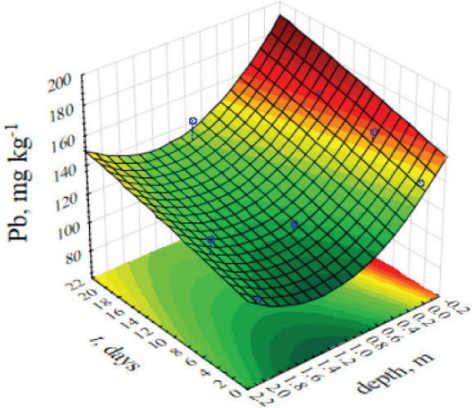

a

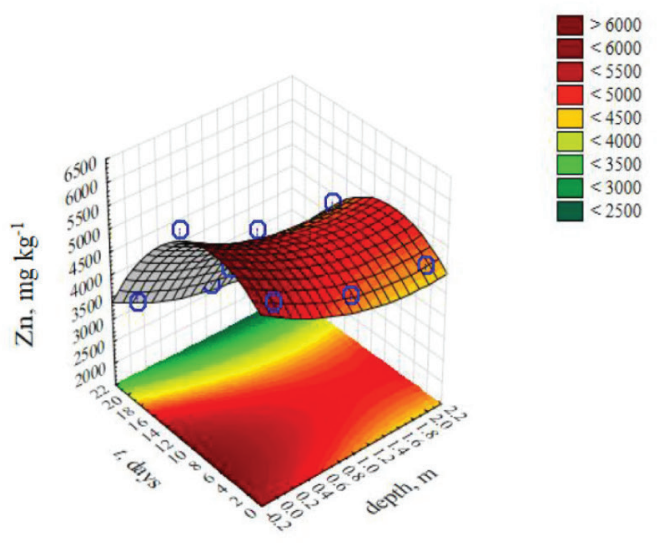

a

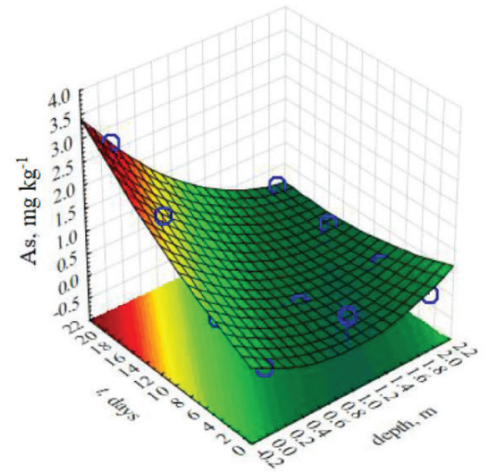

b
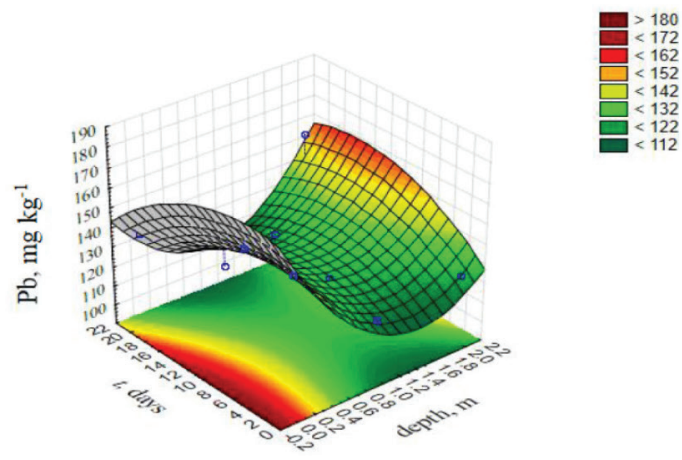

b

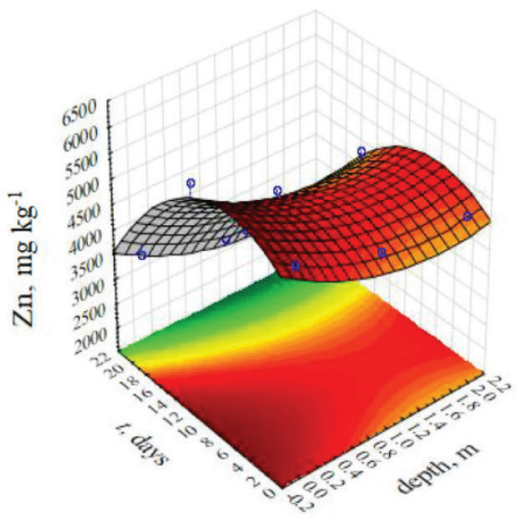

b

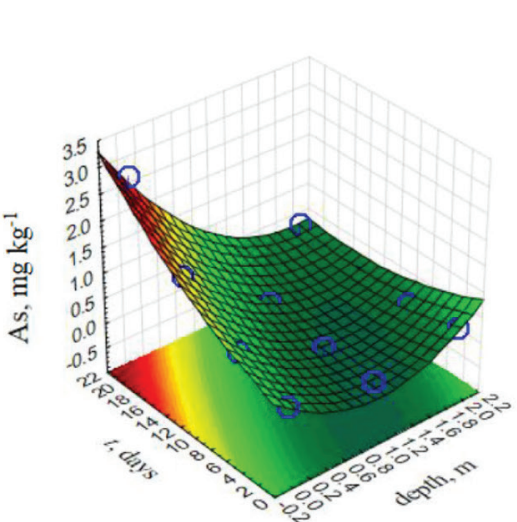

Fig. 3 - Changes in $\mathrm{Cd}, \mathrm{Cu}, \mathrm{Mn}, \mathrm{Ni}, \mathrm{Pb}, \mathrm{Zn}$, and As concentrations with time and depth during: (a) leaching test in deionized water, and (b) leaching test in acid rain

Furthermore, small concentrations were recorded for the following elements in both procedures: $\mathrm{Ag}, \mathrm{Ba}, \mathrm{Co}, \mathrm{Ni}, \mathrm{P}, \mathrm{Sr}, \mathrm{As}$, and $\mathrm{Cr}$. With regard to quality control, the metal content for the blank method were not detected above detection limits. By comparing the two leaching tests, the results showed a slight difference in the concentrations of the analyzed elements.

The major difference was observed in the amount of $\mathrm{Fe}, \mathrm{Si}$, and $\mathrm{S}$, whereas $\mathrm{Ba}, \mathrm{P}$, and $\mathrm{As}$ vary slightly. Fe and $\mathrm{S}$ are more leachable in the synthetic acid rain leaching tests, while $\mathrm{Si}$ is more leachable with deionized water. The concentration of $\mathrm{Cr}$ for days 7 and 14 was below the detection limits of analysis, which means that $\mathrm{Cr}$ was not leachable after the first day. Therefore, the use of acid rain promoted the release of minor and major elements due to acidification. The concentrations of $\mathrm{Zn}, \mathrm{Cd}, \mathrm{Cu}$, and $\mathrm{Pb}$ were high in the beginning, but declined with prolonged leaching. The results of long-term leaching of heavy metals showed that contamination caused by the above elements was serious in the first days, and the trend slowed down with prolonged leaching. Total concentrations of As and heavy metals in jarosite waste were much higher than those evaluated by leaching tests. These results 
were consistent with those reported by Lim et al. ${ }^{17}$ and Yang et al. ${ }^{18}$ Overall, according to the results, the jarosite waste contained certain pollutants which are a heavy burden on the environment. The obtained values for $\mathrm{Zn}, \mathrm{Cd}, \mathrm{Mn}, \mathrm{Cu}, \mathrm{Pb}$, and $\mathrm{As}$ exceed the European regulatory limit for heavy metals in soil ${ }^{14}$. The metal release risk for these metals is still very high due to the low $\mathrm{pH}$ and acid rain. Researchers have predicted that the increase in concentration in the leachate would affect the efficiency of subsequent leachate treatment ${ }^{12,19}$. Heavy metals in jarosite waste will be leached out due to weathering and acid rain, and this cumulative effect of leaching will provide favorable conditions for heavy metals to be leached out.

\section{Statistical analysis}

The quantities of $\mathrm{Cd}, \mathrm{Cu}, \mathrm{Mn}, \mathrm{Ni}, \mathrm{Pb}, \mathrm{Zn}$, and As leached out in both tests are considerably higher than in any previous tests ${ }^{20}$. Three-dimensional surfaces were plotted for the results of the deionized water leaching test (Fig. 3a), and the synthetic acid rain leaching test (Fig. 3b) for the elements with highest impact on the environment.

When comparing the plot of the results of the deionized water leaching test with that obtained for the results of the synthetic acid rain leaching test, it is possible to visualize that the region where the leachates are maximized is not exactly the same in both graphs for each analyzed element. The surfaces presented in Figs. 3, 4, and 5 describe the variations of the leachate amounts as a function of the variations of leaching times and sample depths. In order to visualize a function of two variables (the amount of element leached in deionized water and synthetic acid rain from three-dimensional data presented in Fig. 3, a contour plot for both (a) and (b) graphs was created (Fig. 4). This contour plot shows the level curves of a function of major and minor elements variables from two of the three-dimensional graphs as a function of time and depth. To establish such plot, first the matrix that holds the values of the function was created, and then the contour plot of that matrix. The plotting of a function of two variables such as copper, zinc, lead, manganese, nickel, cadmium, and arsenate are shown in Fig. 4. The overlaying plot attained shows an area where the imposed criteria are satisfied. A point was assigned in this area as the optimization point, which corresponds to the leachant concentration in deionized water and synthetic acid rain leaching conditions. The optimum leaching conditions were performed and the results obtained for metal concentration and the deionized and synthetic acid rain activity of the leachant revealed close agreement with the result predicted by statistical analysis. However, it is worthy emphasizing that, although the two leaching conditions are the most commonly used as leaching solvents, the toxic characteristics of the metals in acid rain conditions raises serious issues when the purpose of the elements leached by these solvents is the application of the material in industrial processes.

In order to verify the effects of each operational variable in the responses, Pareto charts were plotted as well (Fig. 5). In Fig. 5, bars extending to the vertical line corresponded to the effects of $95 \%$ confidence level. As may be seen from Fig. 5, the variable of depth almost exerted a significance level of $p<0.05$ in all the evaluated responses with element concentration in both deionized water and synthetic acid rain. Depth showed to be the most important variable affecting the leaching of major and minor elements under water leaching conditions (Fig. 5a), as well as leaching under acidic conditions (Fig. 5b). All investigated samples (from all depths) are mostly composed of approximately the same phases, yet the concentration can differ based on the Trepca working capacity (Table 1). Time was also an important variable contributing to the leaching of elements such as $\mathrm{Zn}$ and $\mathrm{Ni}$ under synthetic acidic rain conditions. The variable of time exerted a significance level of $p<0.05$ in the evaluated responses with all element concentration in deionized water and for $\mathrm{Zn}$ and As in synthetic acid rain conditions. In general, the Pareto charts allowed observation of both the magnitude and the importance of an effect. These charts displayed the absolute value of the effects, and drew a reference line on the chart. Any effect that extends past this reference line is potentially important. Analysis of the individual factors on the Pareto chart showed that leaching time and depth were statistically significant since they overshot the critical value line (reference line) for almost all analyzed elements, except $\mathrm{Cd}$ and $\mathrm{Cu}$ (Fig. 5b). There was no significant interaction $(\mathrm{L} 1 \times \mathrm{L} 2)$ among the factors, because all the interactions fell short of the critical value line. The interactions for the majority of elements, except As and $\mathrm{Ni}$, do not differ much from both chart distributions. The Pareto charts for major elements $\mathrm{Cd}, \mathrm{Cu}$, $\mathrm{Ni}, \mathrm{Mn}, \mathrm{Pb}, \mathrm{Zn}$, and As are presented in Fig. 5.

Mathematical models describing the response variations ( $t$, days, and depths) as a function of variances in the major and minor element concentration in deionized water and synthetic acid rain were established (Table 4). All models were established with statistically significant terms of $p<0.05$. All mathematical models describing the response variations presented linear (L) and quadratic (Q) terms, as shown in Fig. 4, where both variables of time and depth presented a statistical significance at $p<0.05$. The quadratic term for most of the element concentrations presented the negative signal. This 
$\mathrm{Cd}$

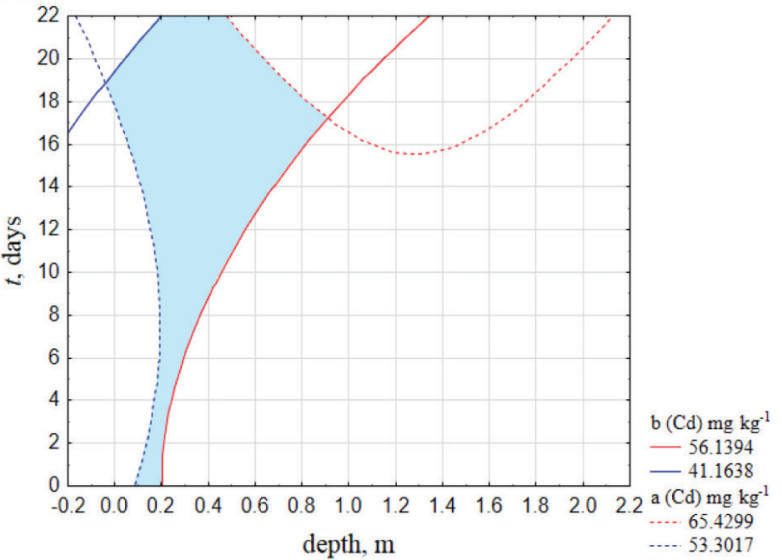

$\mathrm{Mn}$

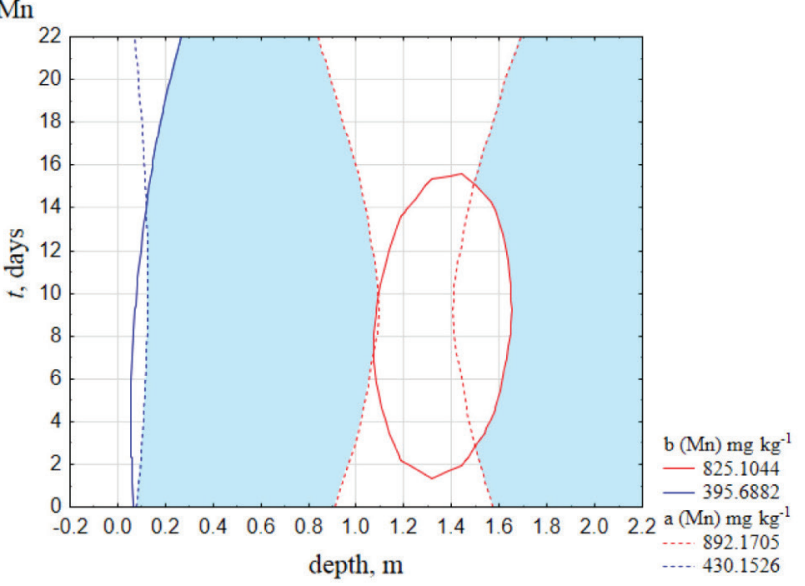

$\mathrm{Pb}$

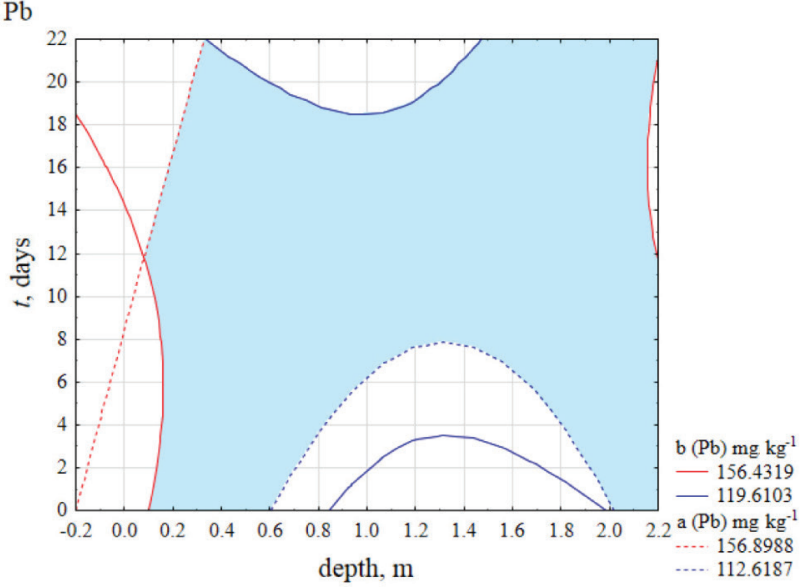

As

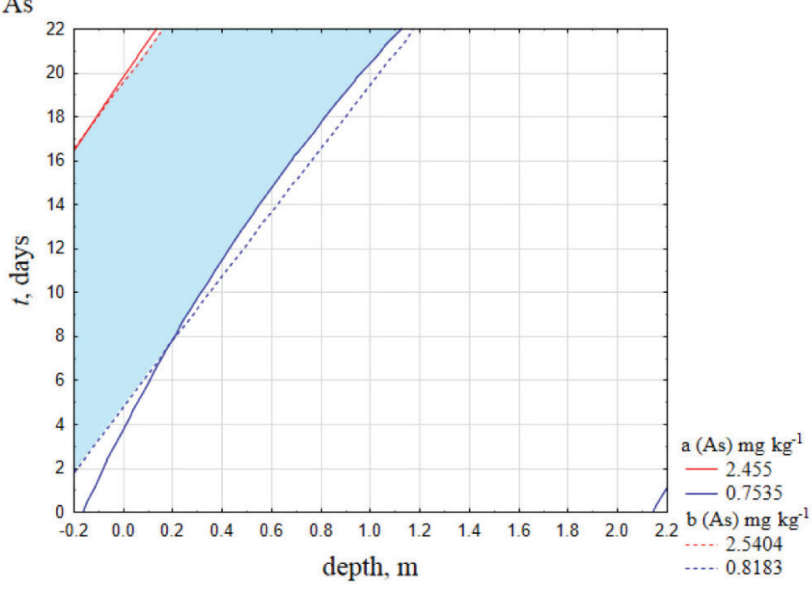

$\mathrm{Cu}$

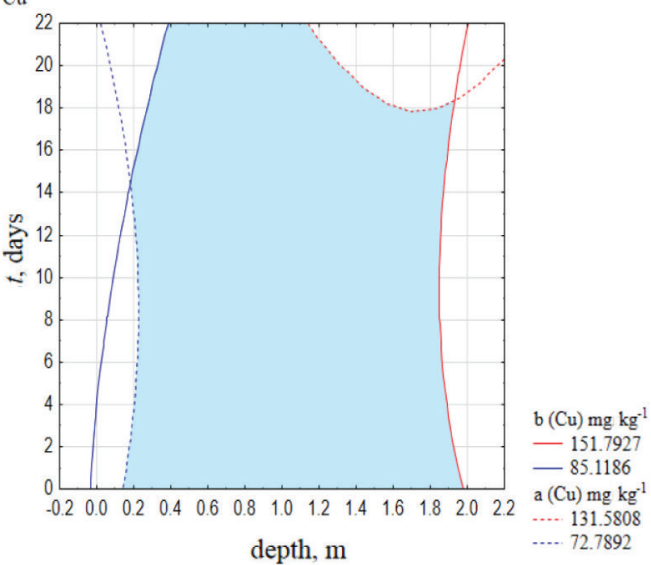

$\mathrm{Ni}$
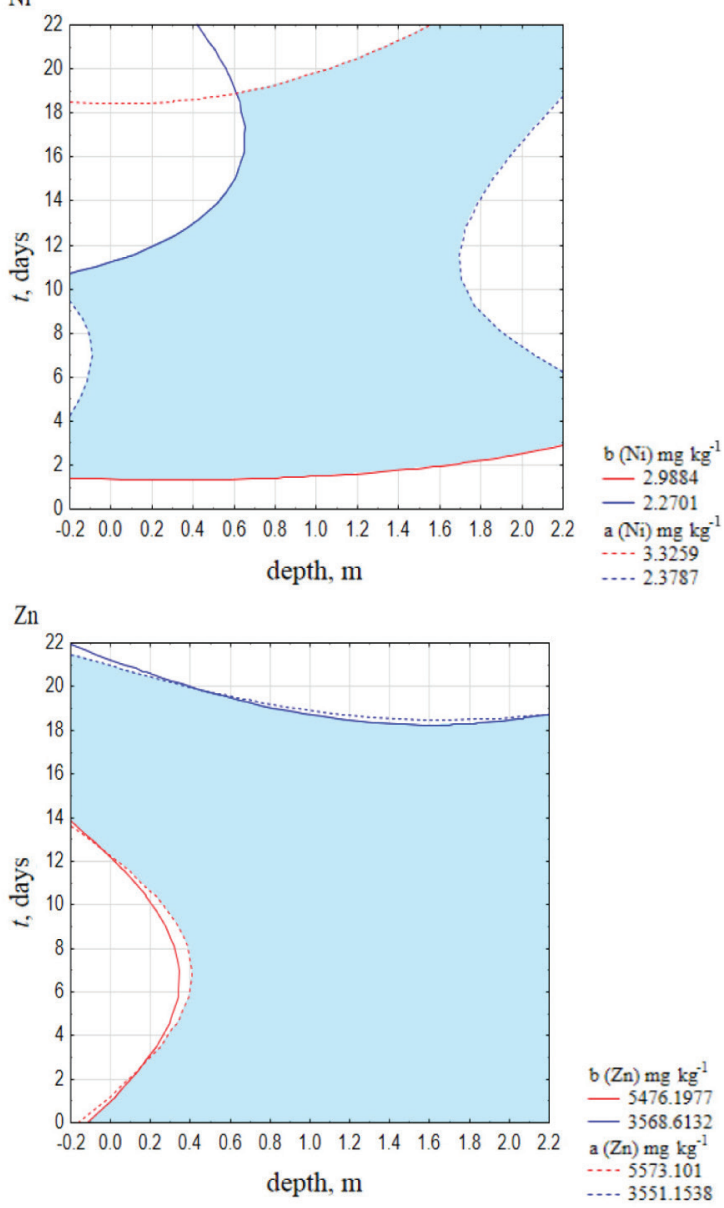

Fig. 4 - The contour plots of element concentration ( $\mathrm{Cd}, \mathrm{Cu}, \mathrm{Mn}, \mathrm{Ni}, \mathrm{Pb}, \mathrm{Zn}$, and $\mathrm{As}$ ) in (a) deionized water, and (b) synthetic acid rain as a function of time ( $t$, days) and depth $(S, m)$ 
a

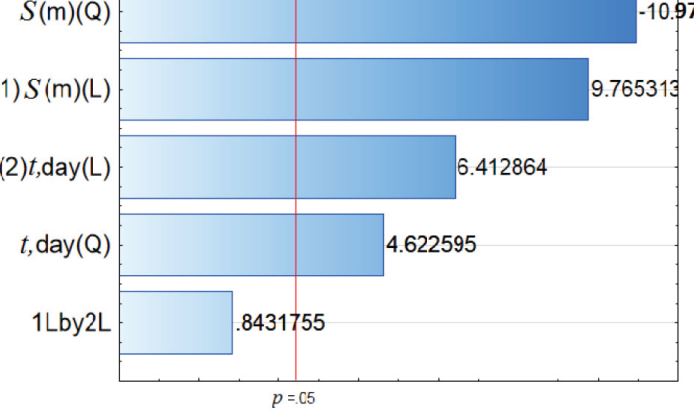

Standardized Effect Estimate (Absolute Value)

a

(1) $S(\mathrm{~m})(\mathrm{L})$

$$
S(\mathrm{~m})(\mathrm{Q})
$$

(2) $t$, $\operatorname{day}(\mathrm{L})$

$t$, day $(Q)$

1Lby2L

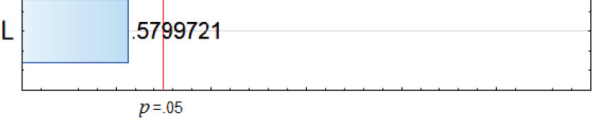

Standardized Effect Estimate (Absolute Value)

a

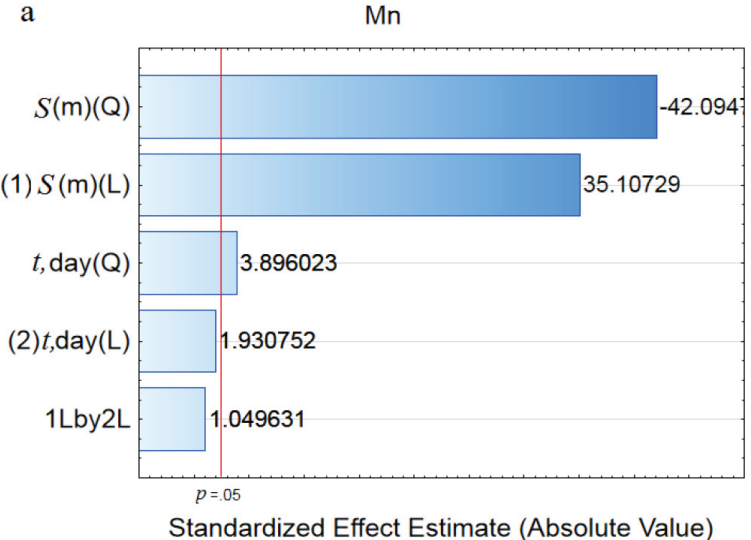

a

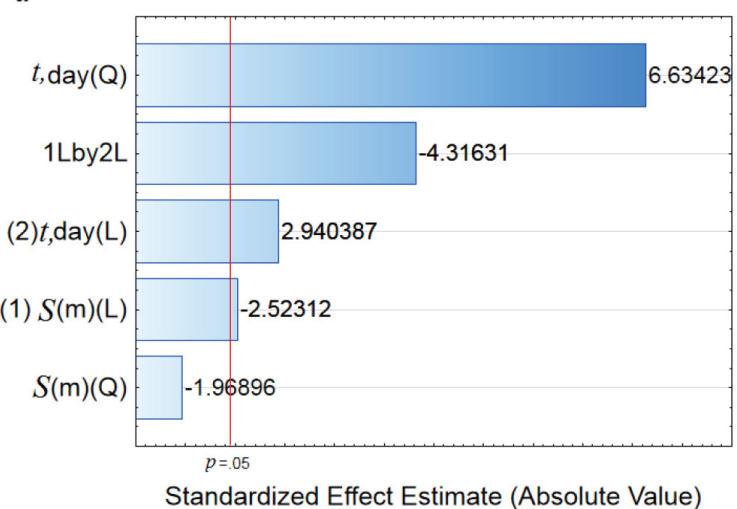

b

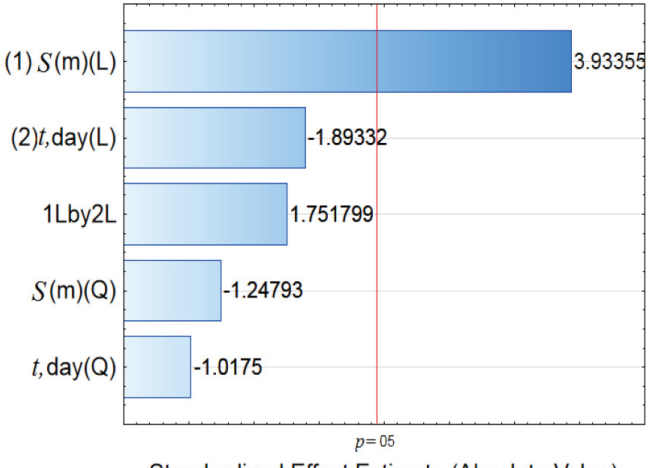

Standardized Effect Estimate (Absolute Value)

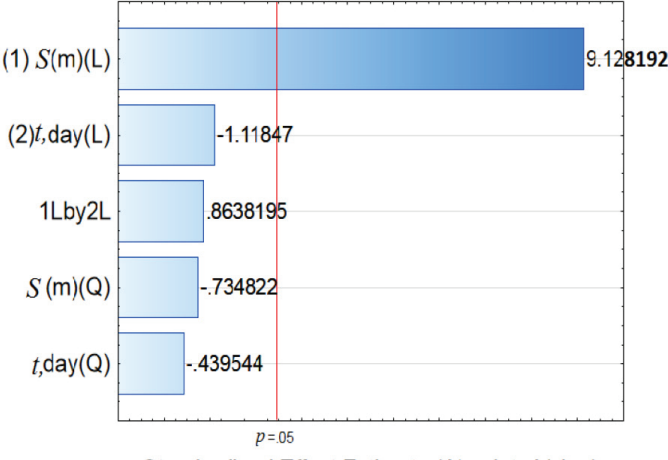

Standardized Effect Estimate (Absolute Value)

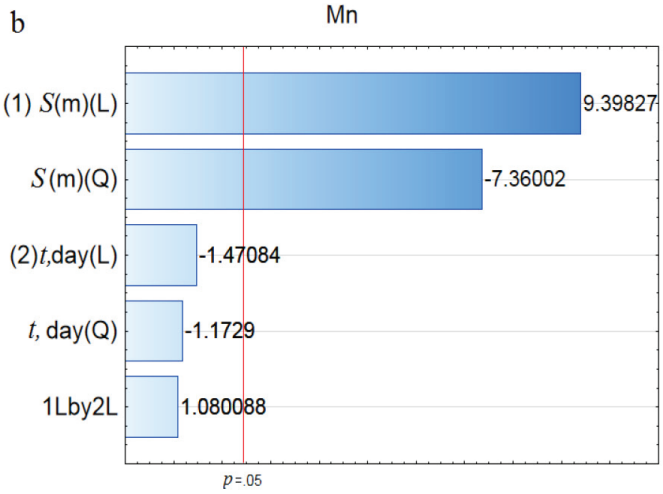

Standardized Effect Estimate (Absolute Value)

b

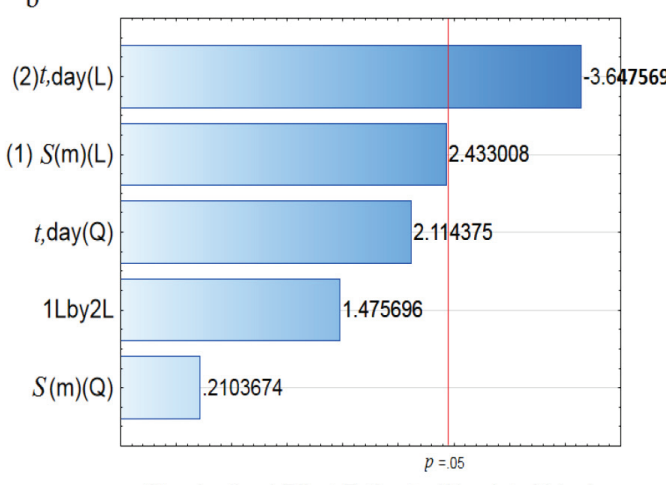

Standardized Effect Estimate (Absolute Value)

Fig. 5 - Continued on next page 
a

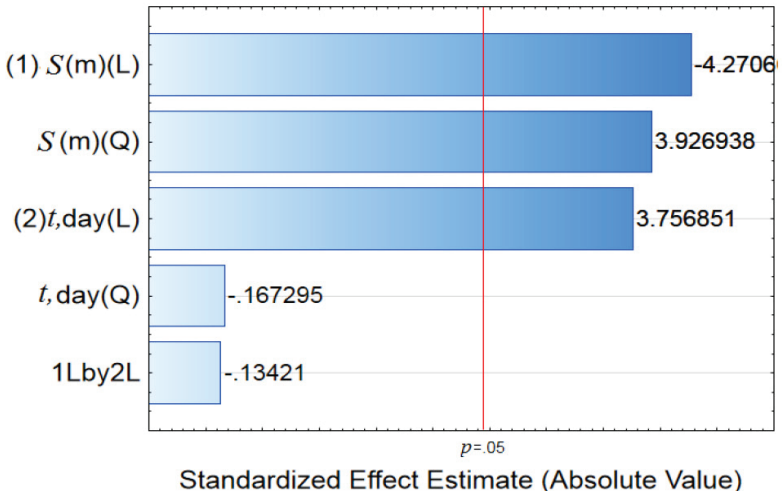

Standardized Effect Estimate (Absolute Value)

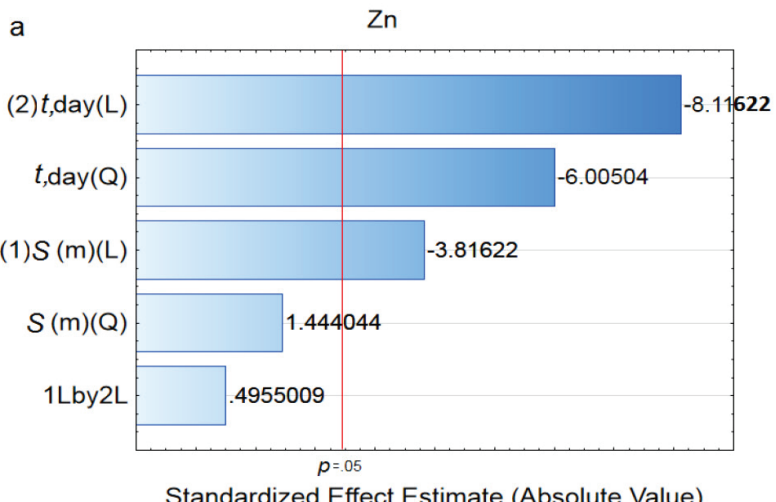

Standardized Effect Estimate (Absolute Value)

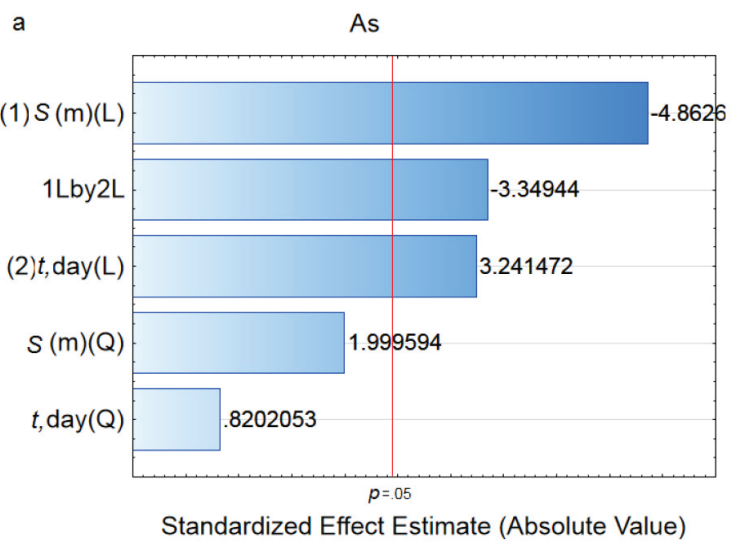

b

$\mathrm{Pb}$

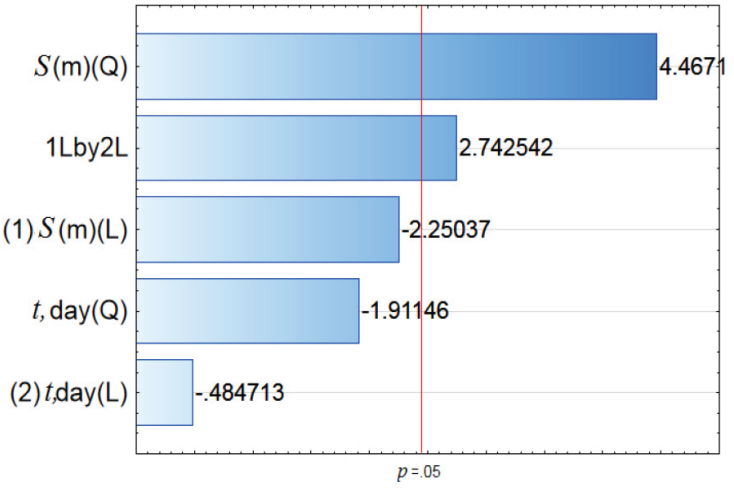

Standardized Effect Estimate (Absolute Value)

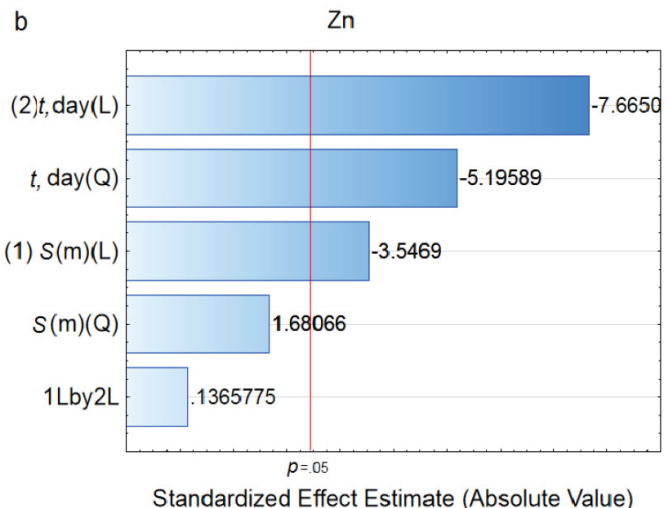

b

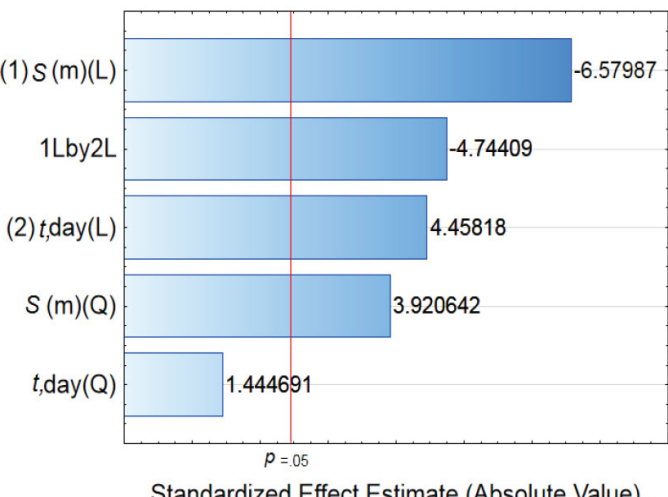

Fig. 5 - Pareto charts for the effects of the element concentration in different leaching time ( $t$, days) and different depths (S, $m$ ), (a) $\mathrm{Cd}, \mathrm{Cu}, \mathrm{Mn}, \mathrm{Ni}, \mathrm{Pb}, \mathrm{Zn}$, and As leaching in deionized water, and (b) $\mathrm{Cd}, \mathrm{Cu}, \mathrm{Mn}, \mathrm{Ni}, \mathrm{Pb}$, Zn, and As leaching in synthetic acid rain

means that the leaching results had not linearly increased when the depth and time had changed, but there was an optimal point after which the use of deionized water and synthetic acid rain did not improve the leaching results. Thus, behaviour was well visualized through the three-dimensional plots presented in Figs. 3a and 3b. However, according to Pareto charts, the linear and quadratic term of time and depth showed to be the most significant for the leaching amount of major and minor elements in jarosite waste. For example, the quadratic term was significant for $\mathrm{Cd}, \mathrm{Ni}$, and $\mathrm{Pb}$, which means that the leaching results had not linearly increased when the different time and depth was analyzed, while the linear term showed to be significantly important for the rest of the analyzed elements.

\section{Conclusions}

Toxicity leaching test revealed high risk from the jarosite waste tailing damps in Mitrovica, Kosovo. The heavy metal concentration in the leachates of jarosite samples was high, particularly that of $\mathrm{Zn}$. 
Table 4 -Polynomial equations fitted to the results of major elements concentration leached in deionized water and synthetic acid rain as leachant

\begin{tabular}{cl}
\hline Response & \multicolumn{1}{c}{ Model equation $^{2}$} \\
\hline $\mathrm{Ni}$ & Leachant: deionized water \\
$\mathrm{Pb}$ & $Z=2.8094+0.6527 x-0.1071 y-0.2085 x^{2}-0.0347 x y+0.0073 y^{2}$ \\
$\mathrm{Zn}$ & $Z=5396.2202-1038.8739 x+159.2697 y+271.9854 x^{2}+7.0783 x y-11.8033 y^{2}$ \\
$\mathrm{As}$ & $Z=0.4536-0.988 x+0.0655 y+0.4901 x^{2}-0.0623 x y+0.0021 y^{2}$ \\
$\mathrm{Cu}$ & $Z=62.383+76.4699 x-1.3803 y-22.6077 x^{2}+0.1127 x y+0.0807 y^{2}$ \\
$\mathrm{Cr}$ & $Z=-0.6714+10.5696 x+16.3784 y-12.3923 x^{2}-2.2132 x y-0.5778 y^{2}$ \\
$\mathrm{Mn}$ & $Z=360.6329+922.6969 x-7.5679 y-371.5122 x^{2}+0.7026 x y+0.3588 y^{2}$ \\
\hline $\mathrm{Ni}$ & Leachant: synthetic acid rain \\
$\mathrm{Pb}$ & $Z=2.8094+0.6527 x-0.1071 y-0.2085 x^{2}-0.0347 x y+0.0073 y^{2}$ \\
$\mathrm{Zn}$ & $Z=163.7002-74.532 x+1.1687 y+26.3478 x^{2}+1.2269 x y-0.1177 y^{2}$ \\
$\mathrm{As}$ & $Z=5350.1932-1063.7322 x+137.1588 y+322.5079 x^{2}+1.9877 x y-10.405 y^{2}$ \\
$\mathrm{Cu}$ & $Z=0.5296-1.2421 x+0.049 y+0.6289 x^{2}-0.0577 x y+0.0024 y^{2}$ \\
$\mathrm{Cr}$ & $Z=86.6615+43.5113 x-0.2468 y-5.3633 x^{2}+0.4782 x y-0.0335 y^{2}$ \\
$\mathrm{Mn}$ & $Z=0.2807+0.0522 x-0.01 y+0.0226 x^{2}-0.0015 x y+0.0005 y^{2}$ \\
\hline
\end{tabular}

The experimental results proved that the amount of leached $\mathrm{Zn}$, as well as other heavy metals, such as $\mathrm{Cd}$ and As, confirms the hazardousness of jarosite waste. This study revealed that the $\mathrm{pH}$ values of these solutions are low. However, the applied leaching tests showed that some of the elements in the jarosite waste were easily released, such as $\mathrm{Zn}, \mathrm{Mn}$, $\mathrm{S}$ and $\mathrm{Cd}$. The low $\mathrm{pH}$ values and high EC values favored the mobility of metal species. According to the statistical analysis, heavy metal release is influenced strongly by depth and time. The Pareto charts showed that depth is the most important variable affecting the leaching of major and minor elements under deionized water and acid leaching conditions. Time was also an important variable contributing to the leaching of elements such as $\mathrm{Zn}$ and Ni under synthetic acidic rain conditions. Mathematical models that were established with statistically significant terms of $p<0.05$ for describing the variations between major elements concentration, leaching time, and depth showed that the quadratic term of time and depth was the most significant model for the leaching amount of major elements in jarosite waste. The RSM described the variations of the response as a function of the variations of the variables in the studied range of values. These variations and relationship were well visualized using the three-dimensional surface plotted for the results of element concentration in regard to time and depth.

\section{References}

1. Kosson, D. S., Van der Sloot, H. A., Sanchez, F., Garrabrants, A. C., An integrated framework for evaluating leaching in waste management and utilization of secondary materials, Environ. Eng. Sci. 19 (2002) 159. doi: doi: https://doi.org/10.1089/109287502760079188

2. Kim, A. G., Hesbach, P., Comparison of fly ash leaching methods, Fuel 88 (2009) 926. doi: https://doi.org/10.1016/j.fuel.2008.11.013

3. Kim, A. G., Kazonich, G., The silicate/non-silicate distribution of metals in fly ash and its effect on solubility, Fuel $\mathbf{8 3}$ (2004) 2285.

doi: https://doi.org/10.1016/j.fuel.2004.06.005

4. EN 12457-2:2002, Characterisation of waste - leaching compliance test for leaching of granular waste materials and sludges - Part 2: one stage batch test at a liquid to solid ratio of $101 / \mathrm{kg}$ for materials with particle size below $4 \mathrm{~mm}$ (without or with size reduction).

5. US EPA, 1992, Toxicity characteristic leaching procedure (TCLP) Method 1311:1992.

6. US EPA, 1994, Synthetic Precipitation Leaching Procedure (SPLP) Method 1312: 1994.

7. US EPA, 2006, Quality Assurance guidance document: Guidance on Systematic Planning Using the Data Quality Objective Process (QA/G-4), EPA/240/B-06/001.

8. Asokan, P., Saxena, M., Asolekar, Sh. R., Hazardous jarosite use in developing non-hazardous product for engineering application, J. Hazard. Mater. 137 (2006) 1589. doi: https://doi.org/10.1016/j.jhazmat.2006.04.054

9. Jegadeesan, G., Al-Abed, S. R., Pinto, P., Influence of trace metal distribution on its leachability from coal fly ash, Fuel 87 (2008) 1887. doi: https://doi.org/10.1016/j.fuel.2007.12.007 
10. Kruger, O., Kalbe, U., Berger, W., Simon, F. G., Meza, S. L., Leaching experiments on the release of heavy metals and $\mathrm{PAH}$ from soil and waste materials, J. Hazard. Mater. 51 (2012) 207.

11. HRN EN, 2005. Croatian Standard on waste characterization, 12457-2.

12. Gitari, W. M., Fatoba, O. O., Petrik, L. F., Leaching characteristics of selected South African fly ashes: Effect of $\mathrm{pH}$ on the release of major and trace species, Journal of Environmental Science and Health, Part A: Toxic/Hazardous Substances and Environmental Engineering 44 (2009) 206. doi: https://doi.org/10.1080/10934520802539897

13. Margui, E., Salvado, V., Queralt, I., Hidalgo, M., Comparison of three-stage sequential extraction and toxicity characteristic leaching tests to evaluate metal mobility in mining wastes, Anal. Chim. Acta. 524 (2004) 151. doi: https://doi.org/10.1016/j.aca.2004.05.043

14. European Council, 2002, Council Decision 2003/33/EC of 19 December 2002 establishing criteria and procedures for the acceptance of waste at landfills pursuant to Article 16 and Annex II to Directive 1999/31/EC, Off. J. Eur. Communities L11.

15. Min, X., Xie, X., Chai, L., Liang, Y., Li, M., Ke, Y., Environmental availability and ecological risk assessment of heavy metals in zinc leaching residue, Trans. Nonferrous Met. Soc. China 23 (2013) 208 doi: https://doi.org/10.1016/S1003-6326(13)62448-6
16. Tsiridis, V., Petala, M., Samaras, P., Kungolos, A., Sakellaropoulos, $G$., Environmental hazard assessment of coal fly ashes using leaching and ecotoxicity tests, Ecotoxicol. Environ. Safety 84 (2012) 212.

doi: https://doi.org/10.1016/j.ecoenv.2012.07.011

17. Lim, M., Han, G. Ch., Ahn, J. W., You, K. S., Kim, H. S., Leachability of arsenic and heavy metals from mine tailings of abandoned metal mines, Int. J. Environ. Res. Public Health 6 (2009) 2865. doi: https://doi.org/10.3390/ijerph6112865

18. Yang, J. S., Lee, J. Y., Baek, K., Kwon, T. S., Choi, J., Extraction behavior of $\mathrm{As}, \mathrm{Pb}$, and $\mathrm{Zn}$ from mine tailings with acid and base solutions, J. Hazard. Mater. 171 (2009) 443. doi: https://doi.org/10.1016/j.jhazmat.2009.06.021

19. Kim, M. J., Ahn, K. H., Jung, Y., Distribution of inorganic species in mine tailings of abandoned mines from Korea Chemosphere 3 (2002) 307. doi: https://doi.org/10.1016/S0045-6535(02)00307-7

20. Kerolli-Mustafa, M., Fajković, H., Rončević, S., Ćurković, $L$., Assessment of metals risks from different depths of jarosite tailing waste of Trepça Zinc Industry, Kosovo based on BCR procedure, J. Geochem. Explor. 148 (2015) 161. doi: https://doi.org/10.1016/j.gexplo.2014.09.001 